\title{
Polarization-Independent High-Speed Switching in a Standard Non-Linear Optical Loop Mirror
}

Mulvad, Hans Christian Hansen; Galili, Michael; Oxenløwe, Leif Katsuo; Clausen, A. T.; Grüner-Nielsen, Lars Erik; Jeppesen, Palle

Published in:

Conference on Optical Fiber communication/National Fiber Optic Engineers Conference, 2008. OFC/NFOEC 2008.

Link to article, DOI:

10.1109/OFC.2008.4528371

Publication date:

2008

Document Version

Publisher's PDF, also known as Version of record

Link back to DTU Orbit

Citation (APA):

Mulvad, H. C. H., Galili, M., Oxenløwe, L. K., Clausen, A. T., Grüner-Nielsen, L. E., \& Jeppesen, P. (2008).

Polarization-Independent High-Speed Switching in a Standard Non-Linear Optical Loop Mirror. In Conference on Optical Fiber communication/National Fiber Optic Engineers Conference, 2008. OFC/NFOEC 2008. (pp. 1-3). IEEE. https://doi.org/10.1109/OFC.2008.4528371

\section{General rights}

Copyright and moral rights for the publications made accessible in the public portal are retained by the authors and/or other copyright owners and it is a condition of accessing publications that users recognise and abide by the legal requirements associated with these rights.

- Users may download and print one copy of any publication from the public portal for the purpose of private study or research.

- You may not further distribute the material or use it for any profit-making activity or commercial gain

- You may freely distribute the URL identifying the publication in the public portal 


\title{
Polarization-Independent High-Speed Switching in a Standard Non-Linear Optical Loop Mirror
}

\author{
H.C. Hansen Mulvad, M. Galili, L.K. Oxenløwe, A.T. Clausen, L. Grüner-Nielsen ${ }^{(*)}$, and P. Jeppesen \\ COM•DTU, Technical University of Denmark, Building 343, DK-2800 Kgs. Lyngby, Denmark \\ (*) OFS Fitel Denmark ApS, Priorparken 680, DK-2605 Brøndby, Denmark \\ E-mail:hchm@com.dtu.dk
}

\begin{abstract}
We introduce a scheme which allows polarization-independent switching in a standard NOLM. Experimental verification is performed by switching $10 \mathrm{Gbit} / \mathrm{s}$ data with $0.2 \mathrm{~dB}$ polarization-dependence and by error-free demultiplexing of polarization-scrambled $320 \mathrm{Gbit} / \mathrm{s}$ OTDM data.

(C)2008 Optical Society of America

OCIS codes: (060.4370) Nonlinear optics, fibers; (060.4510) Optical communications.
\end{abstract}

\section{Introduction}

In optical communication systems, the state of polarization of the transmitted light is in general unknown since it changes randomly over time due to e.g. varying birefringence of fibers. This implies the need for either active polarization stabilisation or polarization-independent operation of the various signal processing components at the network nodes or receivers. All-optical signal processing is required in ultra-high-speed optical networks based on e.g. optical time-division multiplexed (OTDM) signals which are formed by bit-interleaving a number of data-channels at a low base-rate. Here, the bitrate can reach $640 \mathrm{Gbit} / \mathrm{s}$ or above. For such systems, fiber-based switches have a very high potential since their operation is based on fiber non-linearities which have an almost instantaneous response-time [1]. However, these nonlinear effects, such as cross-phase modulation (XPM) and four-wave mixing (FWM), suffer from inherent polarization-dependence, which usually requires polarization control of pump and data signals before the switch. This is the case for the standard non-linear optical loop mirror (NOLM) [2], since it is based on an XPM-induced phase-shift. Several structural modifications of the NOLM have been proposed in order to make it polarization-independent [3-6]. Other examples of polarization-independent fiber-based demultiplexers were based on FWM [7,8], or XPM-induced spectral broadening [9].

In this paper, we demonstrate for the first time that the NOLM in its standard configuration can operate as a polarizationindependent demultiplexer, even at ultra-high bitrates. This requires no structural modifications, and is achieved solely through adjustment of the power-level and the polarization of the control pulses. The basic principle is to cancel the polarization-dependence of the XPM phase shift by careful use of the periodic power transfer function of the NOLM. This principle requires no specific wavelength allocation of data or control pulses, and imposes no limitation on the bandwidth. The concept is experimentally verified both in a simple proof-of-principle experiment where a $10 \mathrm{Gbit} / \mathrm{s}$ data pulse train is switched with only $0.2 \mathrm{~dB}$ residual polarization-dependence, and in a system experiment where a $320 \mathrm{Gbit} / \mathrm{s}$ OTDM data signal, polarization-scrambled at high-speed $(\sim 113 \mathrm{kHz})$, is demultiplexed with error-free performance and low penalty.

\section{Operation principle}

Fig. 1 (a) shows a standard NOLM. It is made from non-polarization-maintaining (non-PM) fibers and components: standard $3 \mathrm{~dB}$ single-mode couplers for coupling the control and data-signals in/out of the loop, a polarization-controller to adjust the net birefringence of the loop, a highly nonlinear fiber (HNLF) as the non-linear medium, and a bandpass-filter (BPF) to filter out the control pulses at the output port. The standard operation of a NOLM is well-known and relies on cross-phase modulation between the high-powered control pulses and the synchronous, clockwise (CW) propagating data-channel pulses [2]. The control-pulse-induced XPM phase-shift on the CW data-pulses causes them to interfere constructively with the counter-CW propagating pulses at the output port, thus resulting in the demultiplexing of this specific channel. The XPMeffect is polarization-dependent, and ideally, the control and data-polarizations are adjusted to linear and parallel states for maximum phase-shift while propagating in a (ideal) fiber with no birefringence. The control pulse power is then adjusted for maximum transmission of the switched pulses.

The polarization-independent NOLM (denoted PI-NOLM), has the same configuration as the Std-NOLM. The operation is based on the following principle, which is also sketched in Fig. 1 (b). The polarization states are shown to the left: the control polarization is set to a linear state along the $y$-axis, and the data is in a random (elliptical) state with field-components $E_{x}$ and $\mathrm{E}_{\mathrm{y}}$ along the $\mathrm{x}$ - and $\mathrm{y}$-axes, respectively. The XPM phase-shift $\Delta \varphi_{\mathrm{y}}$ induced by the control pulse on the parallel $\mathrm{E}_{\mathrm{y}}$-component, is three times larger than the XPM phase-shift $\Delta \varphi_{\mathrm{x}}$ induced on the perpendicular component $\mathrm{E}_{\mathrm{x}}$ [1]. The power transfer function of the NOLM as a function of the phase-shift of the CW-propagating field is shown to the right. It can be seen that there are conditions under which the XPM phase-shifts of the two data-polarization components $\mathrm{E}_{\mathrm{y}}$ and $\mathrm{E}_{\mathrm{x}}, \Delta \varphi_{\mathrm{y}}$ and $\Delta \varphi_{\mathrm{x}}=1 / 3 \Delta \varphi_{\mathrm{y}}$, respectively, give rise to equal transmissions $\mathrm{T}_{\mathrm{y}}$ and $\mathrm{T}_{\mathrm{x}}$. This will result in a polarization-independent switching 
operation, and it occurs at $\left(\Delta \varphi_{x} ; \Delta \varphi_{y}\right)=(\pi / 2 ; 3 \pi / 2)$ and $\left(\Delta \varphi_{x} ; \Delta \varphi_{y}\right)=(\pi ; 3 \pi)$, as shown in Fig. 1 (b), right. These conditions can be achieved by carefully adjusting the average control pulse power $\mathrm{P}_{\text {ctrl }}$, since the XPM-phase shift is proportional to $\mathrm{P}_{\text {ctrl }}$ [1].
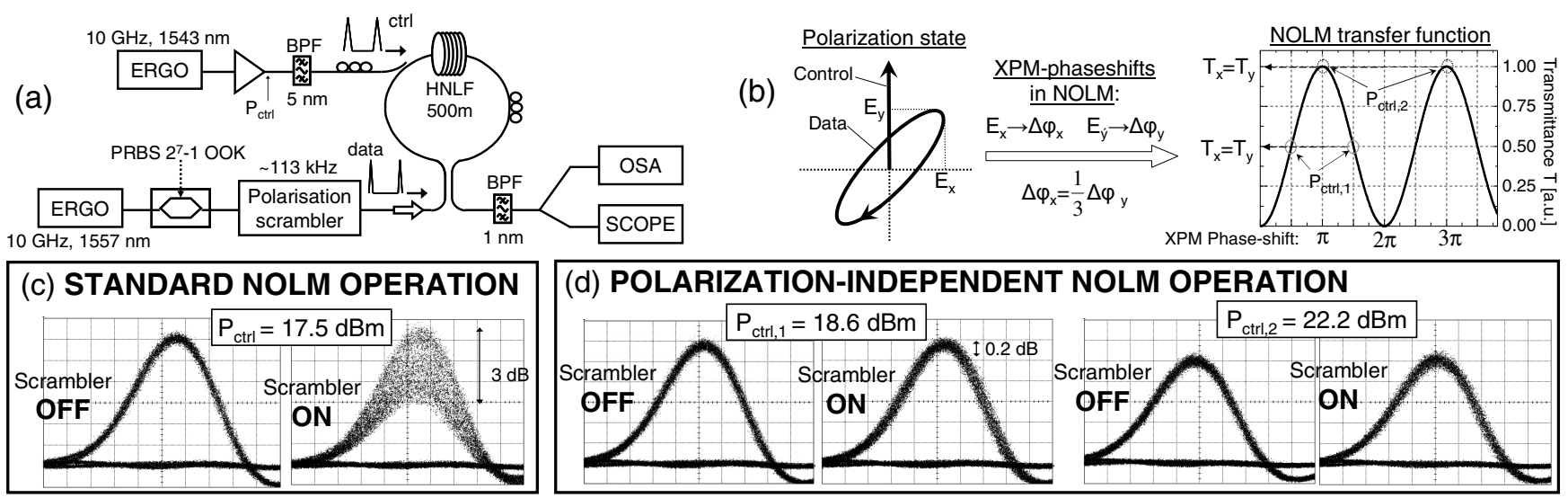

Fig. 1. (a) Experimental set-up for the proof-of-principle experiment: Switching of a $10 \mathrm{Gbit} / \mathrm{s}$ pulse train, (b) Principle behind polarization-independent operation of a standard NOLM, (c) Eye-diagrams of the switched pulse when the NOLM is in standard operation (maximized switched power) with scrambler on/off, (d) Eyes of the switched pulses when the control pulse-power and polarization are adjusted for polarization-independent operation of the NOLM.

\section{Experimental set-up and results}

The set-up for verifying the PI-NOLM principle is shown in Fig. 1 (a). The HNLF in the NOLM is L=500 m long, with a non-linear coefficient $\gamma \sim 10 \mathrm{~W}^{-1} \mathrm{~km}^{-1}$, zero-dispersion wavelength $\lambda_{0}=1550.4 \mathrm{~nm}$ and slope $=0.018 \mathrm{ps} / \mathrm{nm}^{2} \cdot \mathrm{km}$. The $10 \mathrm{Gbit} / \mathrm{s}$ data pulses are obtained from a $10 \mathrm{GHz}$ erbium glass oscillating pulsed laser (ERGO) at a wavelength of $1557 \mathrm{~nm}$, followed by on-off keying (OOK) data-modulation $\left(2^{7}-1\right.$ PRBS) in a Mach-Zehnder modulator. The polarization state of the data signal is then randomized at high speed in a polarization scrambler, where the Poincaré Sphere of polarization states is practically covered by a trajectory which is repeated at a rate of $\sim 113 \mathrm{kHz}$. After the scrambler, the data pulses are injected to the NOLM with an average power of $\sim-3 \mathrm{dBm}$ and a FWHM pulsewidth of $\sim 2 \mathrm{ps}$. The control pulses originate from another $10 \mathrm{GHz}$ ERGO at $1543 \mathrm{~nm}$. They are amplified by a high-power EDFA, filtered by a $5 \mathrm{~nm} \mathrm{BPF}$, and then coupled into the HNLF with a FWHM pulse-width of $\sim 5$ ps. The control pulse polarization state is set by a manual polarization controller.

For the Std-NOLM operation, the control power, data- and control-polarization are adjusted for maximum transmitted data power through the $1 \mathrm{~nm} \mathrm{BPF}$ at the NOLM output. In this case the data-polarization is controlled manually, with the polarization scrambler turned off. The control power is $17.5 \mathrm{dBm}$. The resulting eye diagram is shown in Fig. 1 (c), left. When the scrambler is turned on, the eye is deteriorated as shown in Fig. 1 (c), right. Manually aligning the data-polarization for maximum and minimum switched power yields a polarization-dependent variation of $\sim 3 \mathrm{~dB}$ (measured on the OSA).

For the PI-NOLM operation, the control-power and polarization are modified until the deteriorations induced by the scrambler disappear from the eye-diagram. This occurs at control powers $P_{\text {ctrl }, 1}=18.6 \mathrm{dBm}$ and $P_{\text {ctrl }, 2}=22.2 \mathrm{dBm}$. The corresponding eye-diagrams in Fig. 1 (d) show negligible differences between scrambler on or off. In the case of $\mathrm{P}_{\text {ctrl, }, 1}$, the residual polarization-dependence is measured to be as low as $0.2 \mathrm{~dB}$. This is also the value of the polarization-dependent loss of the NOLM in passive transmission mode (control pulses turned off, constructive interference at the output port). This result confirms that the proposed PI-NOLM can be used to achieve polarization-independent $(<0.2 \mathrm{~dB})$ operation. Precise adjustment of the control power $( \pm 0.1 \mathrm{~dB})$ is required in order to obtain such a low polarization-sensitivity. Furthermore, a constant XPM-phase-shift across the entire data pulse is essential. This can be achieved by using a flat-top control pulse [10], or a Gaussian-like control pulse which is considerably broader than the data pulses, as is the case in this paper.

A high-speed demultiplexing experiment is then conducted in order to test the performance of the PI-NOLM at high bitrates. The experimental set-up is shown in Fig. 2 (a). Compared to the previous set-up, the HNLF is replaced by a $100 \mathrm{~m}$ sample with the same characteristics, and a pulse compressor and OTDM multiplexing stages (MUX) are inserted to generate a $320 \mathrm{Gbit} / \mathrm{s}$ OOK data signal. The compressor is based on self-phase modulation-induced spectral broadening and pulse upchirping in a dispersion-flattened HNLF (DF-HNLF) with negative dispersion. At the input to the HNLF in the NOLM, the FWHM pulse widths are 2.4 ps for the control pulses, and 550 fs for the $320 \mathrm{Gbit} / \mathrm{s}$ data (see autocorrelations in Fig. 2 (e)).

For the Std-NOLM operation, the control power $\mathrm{P}_{\text {ctrl }}$ and polarization are adjusted for the best BER performance of the demultiplexed $10 \mathrm{Gbit} / \mathrm{s}$ data (scrambler off, $\mathrm{P}_{\mathrm{ctrl}}=19.9 \mathrm{dBm}$ ). The resulting eye-diagram is shown in Fig. 2 (b) left, and the corresponding BER curve in Fig. 2 (d) yields a sensitivity (received power for BER $=10^{-9}$ ) of $-34.1 \mathrm{dBm}$. There is no penalty compared to the $10 \mathrm{Gbit} / \mathrm{s}$ back-to-back sensitivity of $-34.0 \mathrm{dBm}$. However, when the scrambler is turned on, the eye is severely deteriorated (Fig. 2 (b), right), and the BER curve exhibits an error-floor above BER=10 $0^{-4}$ (independent of the control 


\section{OMN3.pdf}

polarization). When the control power and polarization are set to achieve polarization-independent operation $\left(\mathrm{P}_{\text {ctrl }}=23.3 \mathrm{dBm}\right)$, the performance is radically improved. The eye-diagrams with scrambler on/off in Fig. 2 (c) show little difference, and the demultiplexed $10 \mathrm{Gbit} / \mathrm{s}$ from the scrambled $320 \mathrm{Gbit} / \mathrm{s}$ is error-free with a sensitivity of $-32.5 \mathrm{dBm}$. When the scrambler is turned off, the sensitivity is $-33.2 \mathrm{dBm}$, which is only a $0.7 \mathrm{~dB}$ difference. BER-measurements are then performed on 14 adjacent channels using the PI-NOLM with scrambler on. They all exhibit error-free performance, and the sensitivities are shown in Fig. 2 (f). These measurements confirm the capability of the proposed PI-NOLM to operate as a polarizationindependent demultiplexer at high bit-rates.

(a) Experimental set-up for $320 \mathrm{Gbit} / \mathrm{s}$ D-MUX

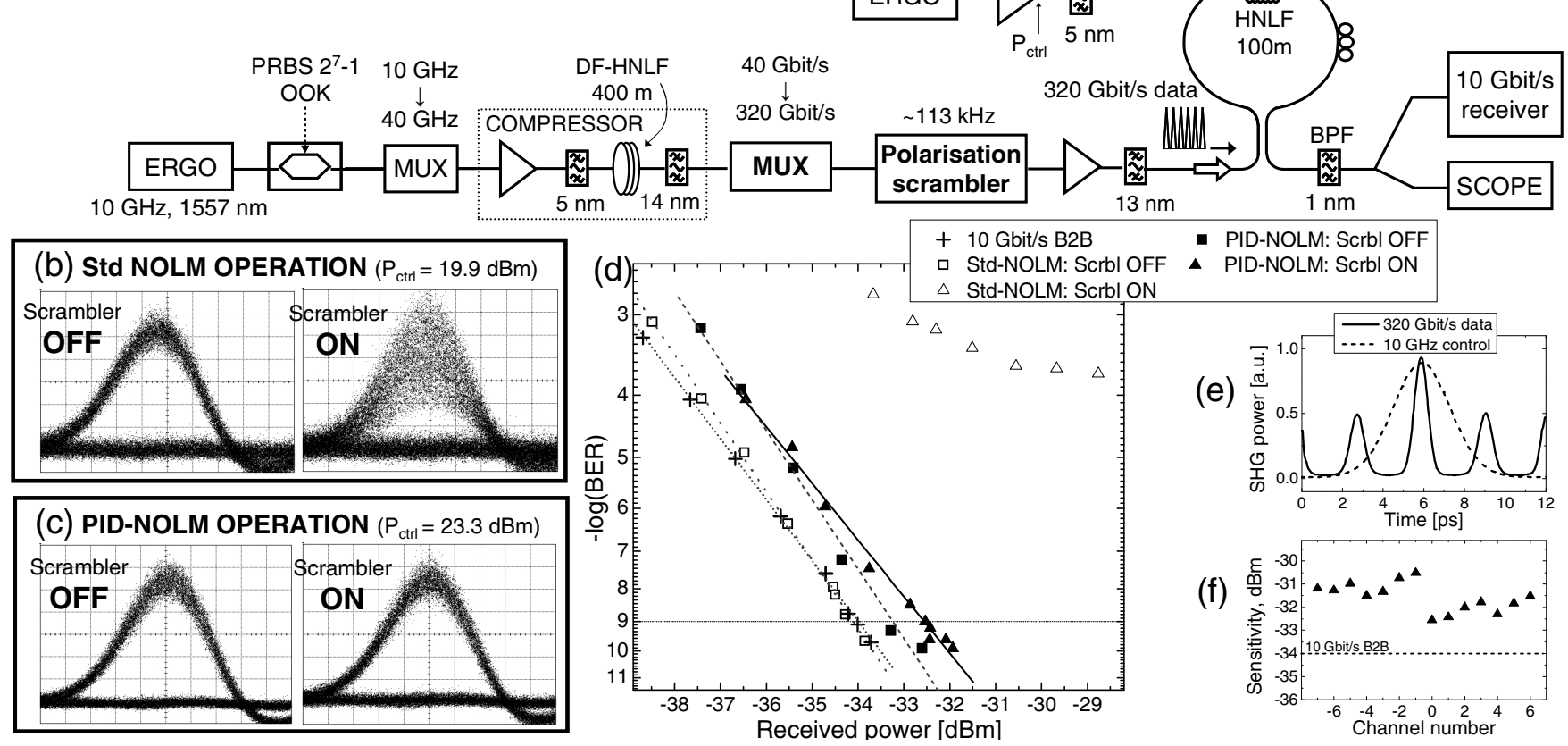

Fig. 2. $320 \mathrm{Gbit} / \mathrm{s}$ polarization-independent demultiplexing. (a) Experimental set-up (b) Eyes of demultiplexed $10 \mathrm{Gbit} / \mathrm{s}$ channel in Std-NOLM operation,

(c) Eyes of demultiplexed $10 \mathrm{Gbit} / \mathrm{s}$ channel in PI-NOLM operation, (d) BER curves, (e) autocorrelations of $320 \mathrm{Gbit} / \mathrm{s}$ data pulses and $10 \mathrm{GHz}$ control pulses, (f) Sensitivities (BER=10-9) of neighbouring channels with PI-NOLM and scrambler on (BER curves for ch. '0' shown in (d)).

\section{Conclusion}

We have proposed and demonstrated a principle which enables polarization-independent operation of a standard NOLM. A polarization-dependence of $<0.2 \mathrm{~dB}$ was demonstrated in a $10 \mathrm{Gbit} / \mathrm{s}$ switching experiment. Furthermore, this method enabled error-free demultiplexing of a polarization-scrambled $320 \mathrm{Gbit} / \mathrm{s}$ OTDM data signal with only $0.7 \mathrm{~dB}$ penalty from the scrambling. With the use of flat-top control pulses [10], we believe that the proposed scheme can be used for polarization-independent demultiplexing at $640 \mathrm{Gbit} / \mathrm{s}$.

\section{References}

[1] G.P. Agrawal, "Nonlinear Fiber Optics", Third edition, Academic Press, 2001.

[2] M. Nakazawa, E. Yoshida, T. Yamamoto, E. Yamada, and A. Sahara, "TDM single channel 640Gbit/s transmission experiment over 60km using 400fs pulse train and walk-off free, dispersion flattened nonlinear optical loop mirror," Electron. Lett., 34, pp. 907-908, 1998.

[3] K. Uchiyama, S. Kawanishi, H. Takara, T. Morioka and M. Saruwatari, "100Gbit/s to 6.3Gbit/s demultiplexing experiment using polarization-independent nonlinear optical loop mirror", Electron. Lett., 30, 11, pp. 873-875, 1994.

[4] B. E. Olsson and P. A. Andrekson, "Polarization independent demultiplexing in a polarization diversity nonlinear optical loop mirror, " IEEE Photon. Technol. Lett., 9, 6, pp. 764 - 766, 1997.

[5] T. Sakamoto, H.C. Lim, and K. Kikuchi, "All-optical polarization-insensitive time-division demultiplexer using a nonlinear optical loop mirror with a pair of short polarization-maintaining fibers", IEEE Photon. Technol. Lett., 14, 12, pp. 1737-1739, 2002.

[6] Z. Wang, L. Huo, C. Lin and C.-K. Chan, "Polarization-independent OTDM demultiplexer based on NOLM with a polarization diversity loop", Optical Fiber Communication Conference, 2006, paper OW114.

[7] T. Morioka, S. Kawanishi, K. Uchiyama, H. Takara and M. Saruwatari, "Polarization-independent 100 Gbit/s all-optical demultiplexer using four-wave mixing in a polarization-maintaining fiber loop", Electron. Lett., 30, 7, pp. 591-592, 1994.

[8] T. Sakamoto, K. Seo, K. Taira, N.S. Moon and K. Kikuchi, "Polarization-insensitive all-optical time-division demultiplexing using a fiber four-wave mixer with a peak-holding optical phase-locked loop", IEEE Photon. Technol. Lett., 16, 2, pp. 563-565, 2004.

[9] R. Salem, A.S. Lenihan, G.M. Carter and T.E. Murphy, "160-Gb/s polarization-independent optical demultiplexing in 2-m nonlinear fiber", IEEE Photon. Technol. Lett., 18, 21, pp. 2245-2247, 2006.

[10] L.K. Oxenløwe, M. Galili, R. Slavík, H.C.H. Mulvad, A.T. Clausen, Y. Park, J. Azaña and P. Jeppesen, "Timing jitter tolerant 640 Gb/s demultiplexing using a long-period fiber grating based flat-top pulse shaper", European Conference on Optical Communication 2007, paper Tu4.5.2. 